\title{
A INFLUÊNCIA DO NÍVEL DE FLEXIBILIDADE E DA MOBILIZAÇÃO ARTICULAR NAS VARIÁVEIS BIOMECÂNICAS DA CORRIDA
}

\author{
THE INFLUENCE OF THE FLEXIBILITY LEVEL AND ARTICULAR \\ MOBILIZATION IN THE BIOMECHANICAL VARIABLES OF THE RUN
}

\section{LA INFLUENCIA DEL NIVEL DE FLEXIBILIDAD Y LA MOVILIZACIÓN ARTICULAR EN LAS VARIABLES BIOMECÁNICAS DE LA CARRERA}

\author{
Hélen Spadari \\ https://orcid.org/0000-0003-2198-3416 (D) \\ http://lattes.cnpq.br/4295913260136461 9 \\ Universidade de Caxias do Sul (Bento Gonçalves, RS - Brasil) \\ helenspadari28@hotmail.com \\ Guilherme Auler Brodt \\ https://orcid.org/0000-0002-8094-9835 if \\ http://lattes.cnpq.br/4124783671364967 \\ Universidade de Caxias do Sul (Caxias do Sul, RS - Brasil) \\ gabrodt@ucs.br
}

\begin{abstract}
Resumo
O objetivo deste estudo foi verificar a influência do nível de flexibilidade e da mobilização articular de membros inferiores nos parâmetros biomecânicos da corrida, sendo categorizado como um estudo experimental com delineamento transversal. Foram avaliados 12 corredores com idade de $33 \pm 3$ anos, sendo 6 mulheres e 6 homens; com o banco de Wells, goniômetro e o sistema de análise de biomecânica cinemática da corrida. Após a primeira coleta foi feita a aplicação da mobilização articular e registrado o resultado do banco de Wells e no sistema de análise 3D. Então foi verificada a relação entre flexibilidade e a mobilização articular com as variáveis biomecânicas da corrida, concluindo que houve correlação de ângulo articular a partir da cinemática nos voluntários com maior amplitude de rotação externa $(0,4555)$ e flexão do quadril $(0,454)$, sendo que no teste de sentar e alcançar houve aumento no escore em centímetros após a intervenção.
\end{abstract}

Palavras-chave: Cinemática; Elasticidade; Resistência; Mobilidade; Lesões.

\begin{abstract}
The purpose of this study is to verify the influence of the level of flexibility and joint mobilization of lower limbs on the biomechanical parameters of running, being categorized as a controlled experimental study with cross-sectional design. Twelve runners, 6 women and 6 men, aged $33 \pm 3$ years were evaluated - with the Wells bench, goniometer and gait analysis system of the UCS. After the first collection, the intervention was performed, which consists of the application of joint mobilization and the result of the Wells bank and the 3D analysis system was recorded. Then, the relation between flexibility and joint mobilization with the biomechanical variables of the race will be verified, concluding that was a correlation of joint angle with the kinematics in volunteers with higher range of external rotation $(0,4555)$ and hip flexion $(0,454)$, being that in the sit and reach test there was an increase in the score in centimeters after the intervention.
\end{abstract}

Keywords: Kinematics; Elasticity; Endurance; Mobility; Injuries.

\section{Resumen}

El objetivo de este estudio es verificar la influencia del nivel de flexibilidad y movilización articular de miembros inferiores en los parámetros biomecánicos de la carrera, siendo categorizado como un estudio experimental de diseño transversal. Se evaluaron 12 corredores, $33 \pm 3$ años, 6 mujeres y 6 hombres, con el banco Wells, goniómetro y el análisis de la marcha de la UCS. Después de la primera recolección, se realizó la intervención, que consiste en la aplicación de la movilización conjunta y se registró el resultado del banco Wells y el sistema de análisis 3D. Luego, 
se verificó la relación entre flexibilidad y movilización articular con las variables biomecánicas de la carrera, concluyendo que hubo correlación del ángulo articular con la cinemática em los voluntarios con mayor rango de rotación externa $(0,4555)$ y flexión de cadera $(0,454)$, siendo que en el test del cajón (alcanzar sientado) hubo un aumento en la puntuación en centímetros después de la intervención.

Palabras clave: Cinemática; Elasticidad; Resistencia; Movilidad; Lesiones.

\section{INTRODUÇÃO}

Assim como em outras modalidades esportivas, a corrida é um meio dinâmico e concreto para desenvolver as pessoas, elevar seus níveis de saúde, promovendo o autoconhecimento, respeito ao próximo, que ensina a aceitar e explorar ao máximo suas possibilidades (RIBEIRO, 2006). Nessas condições houve crescimento no número de praticantes e da quantidade de lesões musculoesqueléticas (ROJO et al., 2017; SCHUBERT, 2014). Desta forma, a ciência vem buscando minimizar tal consequência, sendo a biomecânica uma das áreas que apresenta grande campo para estudo de fatores internos e externos que podem influenciar a ocorrência de lesões (AMADIO; SERRÃO, 2007).

Uma das capacidades físicas que pode ser discutida é a flexibilidade em paralelo à biomecânica, tendo em vista que possui grande influência nas propriedades técnicas dos praticantes e na eficiência dos gestos desportivos (BORGES, 2016). Podendo ser benéfica, quando adequada ou causar instabilidade articular, quando em demasia (PLATONOV, 2003). Sendo que, alguns indícios apontam que a flexibilidade pode estar associada à ocorrência de lesões durante a corrida (NORBERTO; PUGGINA, 2019).

Além da flexibilidade, evidências recentes têm apontado que alterações técnicas na biomecânica da corrida também podem estar relacionadas a ocorrência de lesões como tendinopatia patelar, fascíte plantar, dor patelofemoral e síndrome da banda iliotibial (VANNATTA; HEINERT; KERNOZEK, 2020). Sendo percebidas evidências moderadas de que o aumento da adução do quadril e redução da eversão tornozelos são fatores de risco para lesões relacionadas à corrida. Temos percebido na literatura estudos que apontam intervenções agudas como sendo capazes de alterar os níveis de amplitude de movimento (RUSSO; PONTE, 2011).

Segundo Souza (2016), um importante quadro a ser observado é o padrão de ataque do pé, que pode ser em antepé, mediopé e retropé. O aumento do ângulo de inclinação do pé no contato inicial possui forte correlação com as forças de impacto nas articulações (SOUZA, 2016). Além do ângulo de dorsiflexão durante o contato inicial, o ângulo da tíbia e do joelho também são importantes fatores na mecânica da passada. Ocorre logo depois do 
contato inicial, quando o calçado começa a mudar a direção, são considerados importantes para dissipar o impacto. Outras variáveis de destaque são o pico de flexão do joelho, extensão do quadril e o overstriding, quando há ultrapassagem do pé na frente do centro de massa da pessoa na fase de aterrisagem (SOUZA, 2016). Sobretudo, a literatura carece de informações que façam a associação entre a flexibilidade com variáveis biomecânicas da corrida e se uma intervenção aguda de mobilização articular seria capaz de alterar tais variáveis biomecânicas durante o contato inicial no plano sagital.

Desta forma, o objetivo do presente estudo foi verificar a relação entre o nível de flexibilidade e mobilidade dos membros inferiores (quadril, joelho e tornozelo) com variáveis biomecânicas do contato inicial no plano sagital e avaliar o efeito da mobilização articular nestes parâmetros biomecânicos da corrida.

\section{MÉTODOS}

\section{Participantes}

Para o cálculo amostral foi utilizado o software G*Power 3.1.9.2 (FAUL et al., 2007). No programa como input foram utilizados como referência para as variáveis biomecânicas estudadas um tamanho de efeito de 0,8; probabilidade de erro alfa de 0,05 e; poder estatístico de 0,8 para a família de testes T. Sendo calculado tamanho amostral de 12 corredores. Portanto, como amostra foram recrutadas 6 mulheres ( $34 \pm 5$ anos, $53 \pm 9,5 \mathrm{~kg}$ e $162 \pm 6 \mathrm{~cm}$ ); e 6 homens (32 \pm 3 anos, $70 \pm 6,2 \mathrm{~kg}$ e $175 \pm 8 \mathrm{~cm}$ ) com velocidade média de competição em prova $5 \mathrm{~km}$ de $14 \pm 3 \mathrm{~km} / \mathrm{h}$ (pace aproximado de 5:27 $\mathrm{min} / \mathrm{km}$ a $3: 32 \mathrm{~min} / \mathrm{km}$ ).

Todos os participantes assinaram o termo de consentimento livre e esclarecido, estavam correndo na rua com regularidade de no mínimo três vezes semanais $(20 \mathrm{~km}$ a $80 \mathrm{~km}$ semanais), praticando a modalidade há pelo menos um ano sem interrupções maiores que 15 dias. Se estivessem com dores severas ou moderadas agudas os corredores seriam excluídos, o que não ocorreu com nenhum dos voluntários. O presente trabalho foi aprovado pelo Comitê de Ética em Pesquisa da Universidade de Caxias do Sul. Com a finalidade de caracterizar a amplitude de movimento (ADM) de cada atleta foi realizada Avaliação de ADM completa. Após a Avaliação de ADM, passaram pela avaliação biomecânica antes e após um Protocolo de 
intervenção aguda de mobilização articular. Sendo assim, foi possível verificar os efeitos do protocolo de intervenção aguda de mobilização sobre a biomecânica da corrida.

\section{COLETA DE DADOS}

Avaliação da amplitude de movimento (ADM): para caracterização da ADM de quadril, joelho e tornozelo foram seguidas as recomendações de Marques (2003) e Batista, (2006). Foram avaliados os ângulos máximos de adução, abdução, rotação interna, rotação externa, flexão e extensão de quadris. Extensão e ângulo poplíteo de joelhos. Para tornozelo: Teste Lunge e flexão plantar. Por fim teste de sentar e alcançar no banco de Wells. Totalizando 11 variáveis de desfecho. Apenas a avaliação do banco de Wells foi realizado antes e após a intervenção aguda de mobilização articular.

Avaliação da biomecânica da corrida: Foram aplicados marcadores reflexivos em pontos anatômicos conforme recomendado pelo modelo plugingait (NÜESCH ET AL., 2017). Foi realizado aquecimento em esteira ergométrica (Super ATL - Inbramed Porto Alegre, BR) por 7 a 10 minutos em velocidade confortável até chegar na velocidade de competição referida pelo participante (competição em que apresenta melhor desempenho). Foi mantida a velocidade de competição constante por 10 minutos e então foi capturado o posicionamento tridimensional dos marcadores utilizando sistema de cinemetria de 7 câmeras infravermelho (VICON MX systems, Oxford Metrics Group, UK) a uma taxa de $100 \mathrm{~Hz}$. Trinta segundos foram gravados e 5 passadas completas com sinal consistente foram analisadas. As variáveis cinemáticas de desfecho selecionadas foram os ângulos no contato inicial $(\mathrm{ACl})$ no plano sagital da pelve, quadril, joelho e tornozelo fornecidos pelo software Polygon. Foi realizada coleta da biomecânica da corrida antes e após o protocolo de intervenção aguda de mobilização articular.

Protocolo de intervenção aguda de mobilização articular: Entre as avaliações de corrida e teste foi realizada a intervenção de mobilização articular que consistiu em seis exercícios dinâmicos: dorsiflexão e plantiflexão completa de tornozelos, alternada entre membros em degrau (figura 1A), circundução de cada tornozelo com a ponta dos dedos apoiada sobre o solo (figura 1B), pêndulo em grande ADM de flexo-extensão do quadril no plano sagital (figura 1C) e de abdução-adução no plano coronal (figura 1D), transição do tronco para esquerda e direita no plano coronal a partir da abdução do quadril e extensão do joelho 
ipsilateral e abdução do quadril e flexão do joelho contralateral (figura 1E). Por fim, uma sequência de alongamentos dinâmicos do quadríceps, glúteos e isquiotibiais repetidos 5 vezes para cada membro (figura 1F).

Figura 1 - Sequência de exercícios de mobilização realizados como intervenção do estudo

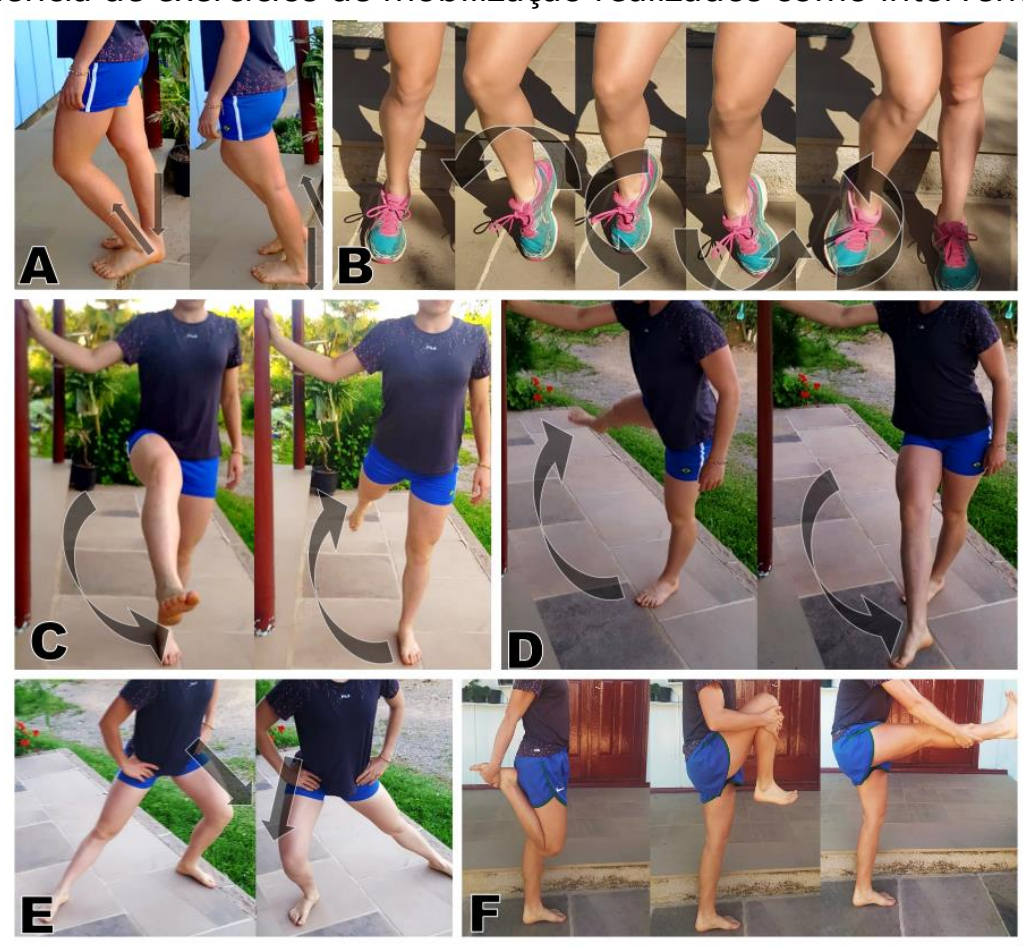

Fonte: acervo dos autores

\section{ANÁLISE ESTATÍSTICA}

Para comparação das variáveis de desfecho antes e depois da intervenção originadas da avaliação biomecânica e de ADM foi utilizado um teste T para medidas repetidas junto ao d de Cohen para cada comparação, após confirmar a normalidade dos dados. Para verificar se existe correlação entre as variáveis de desfecho pré-intervenção foi realizado o teste de correção P de Pearson. Para as variáveis que apresentassem correlação significativa foi elaborado um gráfico com a linha de tendência, equação e $R^{2}$. Todas as análises estatísticas foram realizadas no SPSS 21.0 sendo o alfa adotado menor a 0,05.

\section{RESULTADOS}

As variáveis cinemáticas de desfecho da avaliação biomecânica não apresentaram diferença significativa antes e depois da intervenção. A cadência durante a corrida também parece não sofrer interferências da intervenção de mobilização. A avaliação do banco de Wells 
apresentou aumento médio de 2,9 cm (tabela 1). A tabela 2 apresenta os resultados dos ângulos articulares para fins de descrição das avaliações.

Tabela 1 - Resultados da estatística descritiva e inferencial das variáveis da corrida e banco de Wells avaliadas

\begin{tabular}{lllll}
\hline & Pré & Pós & & \\
Variável & $(\mathrm{M} \pm \mathrm{DP})$ & $(\mathrm{M} \pm \mathrm{DP})$ & $\mathrm{p}$ & $\mathrm{TE}$ \\
\hline ACl Pelve (graus) & $20,34 \pm 5,93$ & $20,11 \pm 5,79$ & 0,901 & $<0,01$ \\
ACl Quadril (graus) & $43,78 \pm 6,28$ & $43,75 \pm 6,44$ & 0,984 & $<0,01$ \\
ACl Joelho (graus) & $10,59 \pm 3,86$ & $10,23 \pm 2,97$ & 0,622 & $<0,01$ \\
ACl Tornozelo (graus) & $6,7 \pm 3,65$ & $6,59 \pm 4,72$ & 0,694 & $<0,01$ \\
Cadência (passos por min) & $173,83 \pm 5,47$ & $173,67 \pm 4,59$ & 0,267 & $<0,01$ \\
Wells (centímetros) & $25,82 \pm 8,34$ & $28,72 \pm 7,25$ & $0,001^{*}$ & 0,399 \\
\hline
\end{tabular}

M: média. DP: desvio padrão. ACl: ângulo no contato inicial. TE tamanho de efeito avaliado pelo d de Cohen

Fonte: construção dos autores

Tabela 2 - Tabela descritiva dos resultados dos ângulos articulares obtidas na avaliação da amplitude de movimento

\begin{tabular}{|c|c|}
\hline Quadril & $M \pm D P\left(I C_{95 \%}\right)$ \\
\hline Abdução de quadril & $67^{\circ} \pm 10^{\circ}\left(47^{\circ}-87^{\circ}\right)$ \\
\hline Rotação Interna & $44^{\circ} \pm 12^{\circ}\left(20^{\circ}-68^{\circ}\right)$ \\
\hline Rotação Externa & $51^{\circ} \pm 11^{\circ}\left(29^{\circ}-73^{\circ}\right)$ \\
\hline Flexão & $121^{\circ} \pm 9^{\circ}\left(103^{\circ}-140^{\circ}\right)$ \\
\hline Extensão & $29^{\circ} \pm 7^{\circ}\left(16^{\circ}-43^{\circ}\right)$ \\
\hline Adução & $50^{\circ} \pm 14^{\circ}\left(22^{\circ}-78^{\circ}\right)$ \\
\hline Joelho & $M \pm D P(I C)$ \\
\hline Flexão & $137^{\circ} \pm 8^{\circ}\left(122^{\circ}-153^{\circ}\right)$ \\
\hline Extensão & $-3^{\circ} \pm 3^{\circ}\left(-10^{\circ}-4^{\circ}\right)$ \\
\hline Angulo Poplíteo & $46^{\circ} \pm 15^{\circ}\left(17^{\circ}-75^{\circ}\right)$ \\
\hline Tornozelo & $M \pm D P(I C)$ \\
\hline Teste Lunge & $41^{\circ} \pm 8^{\circ}\left(26^{\circ}-56^{\circ}\right)$ \\
\hline Flexão plantar & $47^{\circ} \pm 13^{\circ}\left(20^{\circ}-73^{\circ}\right)$ \\
\hline
\end{tabular}

M: média DP: desvio padrão; $I C_{95 \%}$ intervalo de confiança para $95 \%$ dos dados

Fonte: construção dos autores 
Foram encontradas duas correlações significativas entre as variáveis cinemáticas da corrida e as medidas de ADM (tabela 3). Onde os indivíduos com maior mobilidade de rotação externa do quadril apresentavam, no contato inicial, joelho mais flexionado (Figura 2). Os indivíduos que apresentaram o contato inicial em maior dorsiflexão eram os com maior mobilidade em flexão do quadril (Figura 3).

Tabela 3 - Resultados da correlação de Pearson com ângulos articulares e a cinemática da corrida

\begin{tabular}{|c|c|c|c|c|c|c|c|c|c|c|c|}
\hline \multicolumn{12}{|c|}{ Correlações } \\
\hline & $Q$ & Q & $\mathrm{Q}$ & Q & $\mathrm{Q}$ & Q & J & $J$ & J & $\mathrm{T}$ & $\mathrm{T}$ \\
\hline & Abd & $\mathrm{RI}$ & RE & $\mathrm{FL}$ & EXT & ADU & FLEX & EXT & POPL & LUNGE & PLANT \\
\hline \multicolumn{12}{|c|}{ Wells PRÉ } \\
\hline $\mathrm{R}^{2}$ & 0,043 & 0,021 & 0,148 & 0,277 & 0,133 & 0,031 & 0,022 & 0,003 & 0,014 & 0,016 & 0,001 \\
\hline $\mathrm{P}$ & 0,208 & 0,143 & 0,384 & 0,526 & 0,365 & 0,176 & 0,160 & 0,181 & 0,094 & 0,206 & 0,023 \\
\hline Sig & 0,517 & 0,656 & 0,218 & 0,079 & 0,244 & 0,583 & 0,620 & 0,574 & 0,772 & 0,520 & 0,942 \\
\hline \multicolumn{12}{|c|}{$\mathrm{ACl}$ Pelve Pre } \\
\hline$R^{2}$ & 0,006 & 0,048 & 0,032 & 0,018 & 0,001 & 0,000 & 0,281 & 0,000 & 0,011 & 0,055 & 0,110 \\
\hline$P$ & 0,079 & $-0,218$ & 0,179 & 0,134 & $-0,032$ & $-0,004$ & $-0,525$ & $-0,344$ & $-0,151$ & $-0,310$ & $-0,277$ \\
\hline Sig & 0,806 & 0,495 & 0,578 & 0,679 & 0,922 & 0,990 & 0,079 & 0,273 & 0,640 & 0,326 & 0,384 \\
\hline \multicolumn{12}{|c|}{ ACI Quadril Pre } \\
\hline $\mathrm{R}^{2}$ & 0,048 & 0,043 & 0,021 & 0,073 & 0,027 & 0,038 & 0,144 & 0,002 & 0,013 & 0,002 & 0,103 \\
\hline$P$ & $-0,219$ & $-0,208$ & 0,144 & 0,270 & $-0,164$ & $-0,196$ & $-0,385$ & $-0,324$ & $-0,083$ & $-0,111$ & $-0,291$ \\
\hline Sig & 0,495 & 0,517 & 0,655 & 0,396 & 0,610 & 0,542 & 0,216 & 0,304 & 0,798 & 0,730 & 0,359 \\
\hline \multicolumn{12}{|c|}{ ACl Joelho Pre } \\
\hline$R^{2}$ & 0,193 & 0,072 & 0,456 & 0,033 & 0,037 & 0,179 & 0,157 & 0,027 & 0,011 & 0,068 & 0,054 \\
\hline$P$ & $-0,439$ & 0,268 &,$- 675^{*}$ & 0,183 & $-0,193$ & 0,423 & 0,347 & $-0,173$ & 0,200 & 0,178 & 0,064 \\
\hline Sig & 0,153 & 0,400 & 0,016 & 0,570 & 0,548 & 0,171 & 0,270 & 0,591 & 0,534 & 0,579 & 0,844 \\
\hline \multicolumn{12}{|c|}{$\mathrm{ACl}$ Torn. Pre } \\
\hline $\mathrm{R}^{2}$ & 0,019 & 0,083 & 0,046 & 0,454 & 0,160 & 0,008 & 0,321 & 0,583 & 0,642 & 0,027 & 0,009 \\
\hline $\mathrm{P}$ & 0,139 & 0,287 & 0,214 &, $674^{*}$ & 0,400 & 0,088 & 0,521 & $-0,093$ & $-0,049$ & 0,383 & 0,429 \\
\hline Sig & 0,666 & 0,365 & 0,503 & 0,016 & 0,197 & 0,785 & 0,082 & 0,773 & 0,880 & 0,219 & 0,164 \\
\hline \multicolumn{12}{|c|}{ CAD Pre } \\
\hline $\mathrm{R}^{2}$ & 0,006 & 0,085 & 0,199 & 0,035 & 0,225 & 0,008 & 0,001 & 0,058 & 0,045 & 0,048 & 0,072 \\
\hline$P$ & $-0,078$ & 0,292 & $-0,446$ & $-0,186$ & $-0,474$ & $-0,087$ & $-0,082$ & $-0,039$ & 0,124 & $-0,454$ & 0,048 \\
\hline Sig & 0,810 & 0,357 & 0,146 & 0,562 & 0,119 & 0,787 & 0,800 & 0,904 & 0,702 & 0,138 & 0,883 \\
\hline
\end{tabular}

$\mathrm{P}$ indica o valor P de Pearson encontrado. ACl: Ângulo no contato inicial / CAD: cadência / Pre: pré-intervenção / Torn: tornozelo / Q_Abd: abdução do quadril / Q_RI: rotação interna do quadril / Q_RE: rotação externa do quadril / Q_FL: flexão do quadril / QEXT: extensão do quadril / QADU: adução de quadril / JFLEX: flexão de joelho / JEXT: extensão de joelho / JPOPL: ângulo poplíteo do joelho / T_LUNGE: Lunge Test para tornozelo / T_PLANT: flexão plantar

Fonte: construção dos autores 
Figura 2 - Correlação entre ângulo de rotação externa do quadril com o ângulo de flexão do joelho no contato inicial da corrida

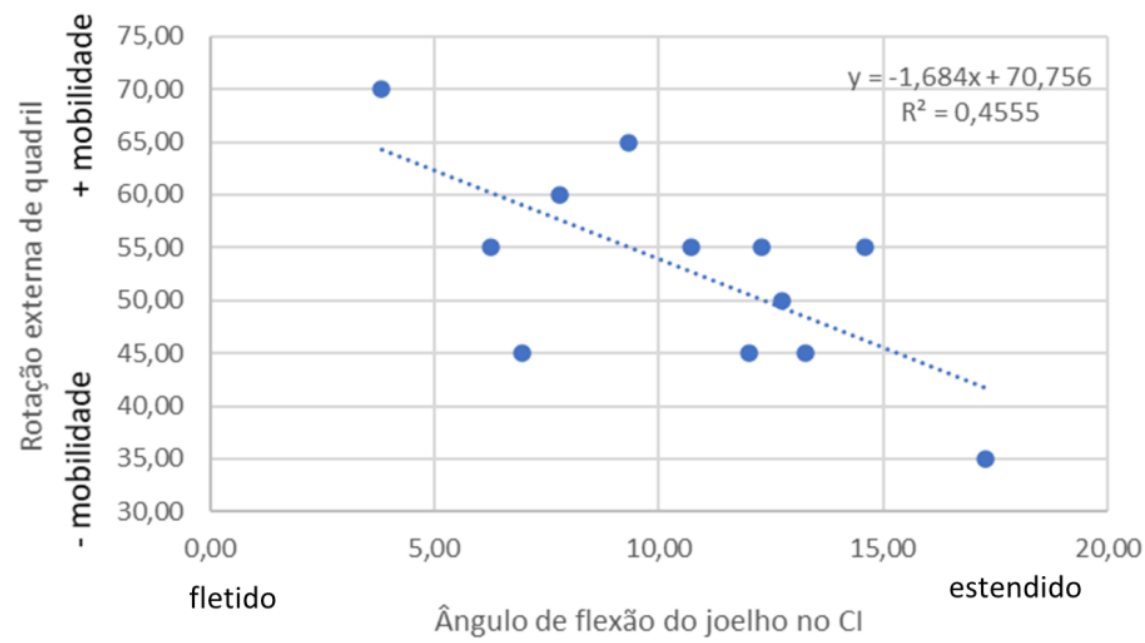

Fonte: construção dos autores

Figura 3 - Correlação entre o ângulo de flexão do quadril com o ângulo do tornozelo no contato inicial $(\mathrm{Cl})$ da corrida

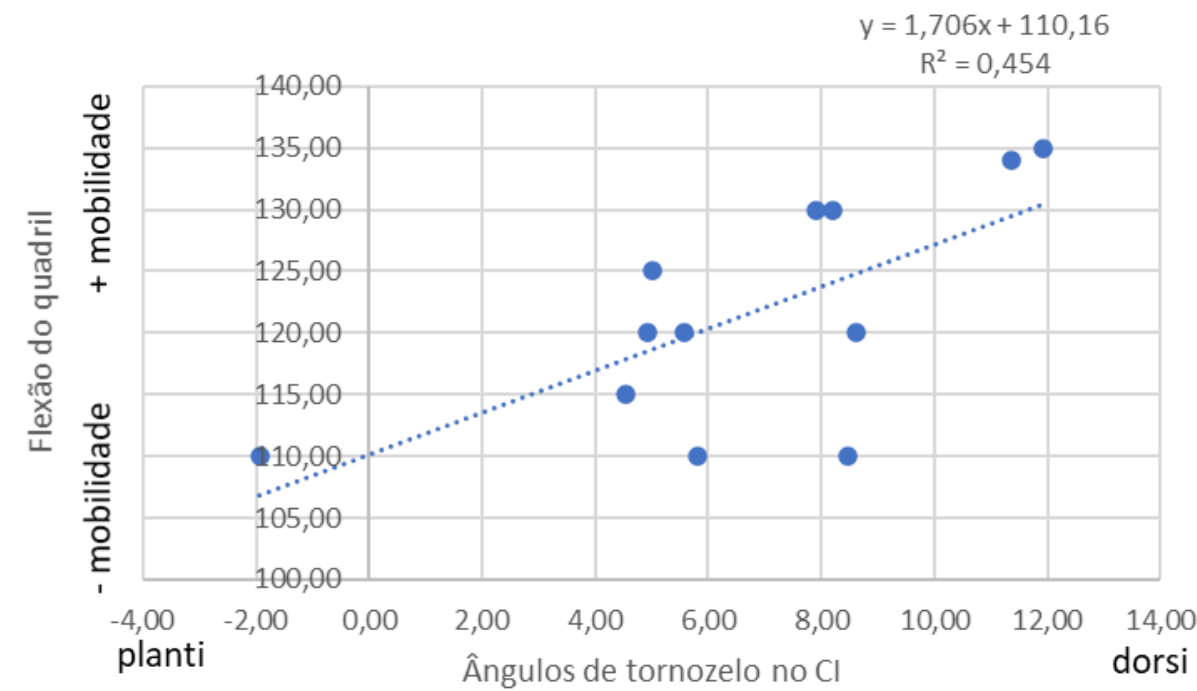

Fonte: construção dos autores

\section{DISCUSSÃo}

Uma capacidade que é discutida na área da corrida é a flexibilidade. Em qual momento do treinamento seria mais eficiente, se esta capacidade realmente é influente no desempenho e de que forma pode ser útil, tanto no gerenciamento de uma lesão e/ou na otimização do movimento. Borges (2016) enfatiza que a flexibilidade possui grande influência nas propriedades técnicas dos praticantes e na eficiência dos gestos desportivos, visto que é 
determinada pela execução de uma atividade em grau de mobilidade máxima do músculo e da articulação.

Apesar de não poder concluir diretamente relação sobre a causa e efeito, podemos aceitar o coeficiente de correlação ao quadrado (conhecido como o coeficiente de determinação, r2) como uma medida da quantidade de variação em uma variável que é explicada pela outra. Então, o r2 pode nos dizer o quanto de variabilidade ocorre (FIELD, 2009) entre variáveis. Desta forma, pode-se dizer que a ADM de rotação externa do quadril é capaz de explicar cerca de $45 \%$ da variação do ângulo de flexão do joelho no contato inicial. Bem como, a ADM de flexão do quadril foi capaz de explicar cerca de $45 \%$ da variação do ângulo do tornozelo no contato inicial.

A relação existente entre a flexibilidade e a técnica de corrida pode estar relacionada a diminuição ao aumento do relaxamento muscular e a influência que a mobilidade articular tem capacidade de produção de força e potência (MONTEIRO, 2006). Além disso, maiores níveis de flexibilidade podem auxiliar na economia de energia durante tarefas como a corrida, já que há relação entre potencial elástico muscular - capacidade de armazenar e liberar energia - com o comprimento dos fascículos e a unidade musculotendínea (JUNIOR, 2007; BORGES, 2016).

Pileggi e colaboradores (2010) apontam para a deficiência de flexibilidade articular como um fator para predisposição de lesões. Silva e Jube (2020) concluem que ganhos de flexibilidade podem influenciar na diminuição do gasto energético e na proteção das estruturas sobrecarregadas durante a corrida. No presente estudo, não foi possível verificar efeitos de uma intervenção aguda nas medidas de amplitude de movimento, com exceção da medida do Banco de Wells, isso se deve provavelmente ao fato do banco de Wells ser um teste que envolve múltiplas cadeias musculares posteriores, que vão desde a panturrilha até os extensores de tronco. Essa grande abrangência muscular desse teste talvez o torne sensível a questões como o aquecimento do corpo causado durante o teste de corrida ou às mobilizações realizadas no estudo em detrimento às demais medições de ADM. Concordando com Rosa e Montandon (2006) que relata o uso do aquecimento antes do alongamento, pois acredita-se que o aumento da temperatura dentro do músculo gera mais plasticidade da musculatura e tendões, por consequência, aumentando a amplitude de movimento e diminuindo riscos de lesões musculares. De um ponto de vista descritivo, se pode notar pelos valores alcançados pelos voluntários no teste de sentar e alcançar e ângulos articulares é que 
de maneira geral, os participantes possuem valores dentro da normalidade (MARQUES, 2003; RIBEIRO, 2010).

Os indivíduos com maior mobilidade de flexão de quadril apresentaram maior dorsiflexão, já os indivíduos com menor mobilidade tiveram maior plantiflexão durante o contato inicial da corrida. A maior mobilidade na flexão do quadril sugere a existência de maior flexibilidade da cadeia posterior, o que parece ter associação com a dorsiflexão no tornozelo durante a corrida. Ou seja, os indivíduos que apresentaram valores menores de flexão do quadril, possuem uma descarga de peso na parte anterior (antepé) do pé associada à diminuição de flexibilidade da cadeia posterior do membro inferior, conduzindo a menores ângulos de dorsiflexão do tornozelo. Apesar de não ser possível estabelecer relação entre causa e efeito, treinadores podem utilizar essa informação para utilizar ganhos de mobilidade de quadril como ferramenta para ajustes na técnica da pisada durante a corrida. Um mecanismo que poderia explicar esse resultado seria a presença de uma "pré-tensão" passiva em tecidos antagonistas da articulação do quadril (SOUZA, 2012). Essa "pré-tensão" pode ter causado uma espécie de frenagem da articulação do quadril levando o contato inicial a acontecer em antepé (maior plantiflexão). Ou o contrário também pode ser verdadeiro: indivíduos que tem o hábito de correr em antepé podem ter desenvolvido maior tensão na articulação do quadril devido a essa estratégia de corrida. Sugerem-se estudos que avaliem o desenvolvimento crônico de mobilidade de quadril para verificar a existência de efeitos a longo prazo na articulação do tornozelo e sua relação entre causa e efeito.

Já os resultados evidenciados nos graus de extensão joelho no contato inicial da corrida, indica que pode ocorrer overstriding - quando há ultrapassagem do pé na frente do centro de massa da pessoa devido ao aumento da extensão do joelho (SOUZA, 2016) fenômeno este que ocorre devido ao joelho exageradamente estendido durante o contato inicial. Essa diminuição da flexão joelho no contato inicial que pode estar relacionada com o overstring, foi percebido nos indivíduos que possuem menor mobilidade de rotação externa do quadril. Os músculos rotadores externos de quadril são importantes estabilizadores durante a corrida, talvez a falta de mobilidade em rotação externa indique um déficit de controle dessa musculatura e/ou articulação, o que levaria a uma corrida com o joelho mais estendido. Quando corremos com os joelhos mais estendidos diminuímos a sobrecarga no quadril e aumentamos no tornozelo (LIEBERMAN, 2015). Com isso, propor intervenções de força e 
mobilidade de quadríceps e rotadores externos do quadril pode ser algo a ser experimentado no âmbito da preparação física desses atletas.

Sugerimos para estudos futuros que as variáveis cinemáticas e ADM devem ser juntamente com histórico de lesões investigadas para possíveis associações. Além disso o efeito crônico de intervenções de flexibilidade também pode ser algo capaz de causar alterações na cinemática da corrida no plano sagital.

\section{CONCLUSÃO}

As variáveis cinemáticas de ângulo articular da pelve, quadril, joelho e tornozelo durante o contato inicial da corrida não apresentaram diferença significativa após a intervenção aguda de mobilização. A cadência durante a corrida também parece não sofrer interferências da intervenção de mobilização. Os indivíduos com maior mobilidade de rotação externa do quadril apresentaram contato inicial com joelho mais flexionado e os indivíduos com maior mobilidade em flexão do quadril apresentaram contato inicial com maior dorsiflexão.

\section{REFERÊNCIAS BIBLIOGRÁFICAS}

AMADIO, Alberto Carlos; SERRÃO, Júlio Cerca. Contextualização da biomecânica para a investigação do movimento: fundamentos, métodos e aplicações para análise da técnica esportiva. Revista brasileira de educação física e esporte, v. 21, p. 61-85, 2007.

BATISTA, Lucia Helena e colaboradores. Avaliação da amplitude articular do joelho: correlação entre as medidas realizadas com o goniômetro universal e no dinamômetro isocinético. Brazilian journal of physical therapy, v. 10, n. 2, p. 193-198, 2006.

BORGES, José Manuel. Teoria e metodologia do treino desportivo: modalidades individuais. Lisboa, Portugal: Instituto Português do Desporto e Juventude, 2016.

FAUL, Franz e colaboradores. G* Power 3: A flexible statistical power analysis program for the social, behavioral, and biomedical sciences. Behavior research methods, v. 39, n. 2, p. 175191, 2007.

FIELD, Andy. Descobrindo a estatística usando o SPSS. 2. ed. Porto Alegre, RS: Artmed, 2009.

JUNIOR, Abdallah Achour. Alongamento e flexibilidade: definições e contraposições. Revista brasileira de atividade física \& saúde, v. 12, n. 1, p. 54-58, 2007. 
LIEBERMAN, Daniel E. e colaboradores. Effects of stride frequency and foot position at landing on braking force, hip torque, impact peak force and the metabolic cost of running in humans. Journal of experimental biology, v. 218, n. 21, p. 3406-3414, 2015.

MARQUES, Amélia Pasqual. Manual de goniômetria. 2. ed. Barueri, SP: Manole, 2003.

MONTEIRO, Gizele de Assis. Treinamento da flexibilidade: sua aplicabilidade para saúde. Londrina, PR: Midiograf, 2006.

NÜESCH, Corina e colaboradores. Measuring joint kinematics of treadmill walking and running: comparison between an inertial sensor based system and a camera-based system. Journal of biomechanics, v. 57, p. 32-38, 2017.

NORBERTO, Matheus Silva; PUGGINA, Enrico Fuini. Relações entre flexibilidade de membros inferiores e índice de lesões em modalidades de resistência. Revista brasileira de ciências do esporte, v. 41, n. 3, p. 290-297, 2019.

PILEGGI, Paula e colaboradores. Incidência e fatores de risco de lesões osteomioarticulares em corredores: um estudo de coorte prospectivo. Revista brasileira de educação física e esporte, v. 24, n. 4, p. 453-462, 2010.

PLATONOV, Vladimir Nicolaievitch; BULATOVA, Marina M. A preparação física. Rio de Janeiro: Sprint, 2003.

RANGEL, Gabriel Mamoru Masuda; FARIAS, Joni Márcio de. Incidência de lesões em praticantes de corrida de rua no município de criciúma, Brasil. Revista brasileira de medicina do esporte, v. 22 , n. 6, p. $496-500,2016$.

RIBEIRO, Cibele Calvi Anic e colaboradores. Nível de flexibilidade obtida pelo teste de sentar e alcançar a partir de estudo realizado na Grande São Paulo. Revista brasileira cineantropometria desempenho humano, v. 12, n. 6, p. 415-421, 2010.

RIBEIRO, Nuno Cobra. A semente da vitória. 84. ed. São Paulo: Senac, 2006.

ROJO, Jeferson Roberto e colaboradores. Transformações no modelo de corridas de rua no Brasil: um estudo na Prova Rústica Tiradentes. Revista brasileira de ciência e movimento, v. 25, n.1, p. 19-28, 2017.

ROSA, Alexandre Carlos; MONTANDON, Ivana. Efeitos do aquecimento sobre a amplitude de movimento: uma revisão crítica. Revista brasileira de ciência e movimento, v. 14, n. 2, p. 103$110,2008$.

RUSSO, André Faria; ADAIL, Karla Paula; PONTE, Maria Glesilene. A eficácia do alongamento ativo comparado ao ultrassom terapêutico no ganho de flexibilidade aguda dos músculos isquiotibiais. Fisioterapia Ser, v. 6, p. 4-8, 2011. 
SCHUBERT, Amy G.; KEMPF, Jenny; HEIDERSCHEIT, Bryan C. Influence of stride frequency and length on running mechanics: a systematic review. Sports health, v. 6, n. 3, mai./ jun., 2014.

SILVA, Renata Cristina Araujo; JUBE, Lucas Paranaiba Medeiros. Os benefícios da prática do método pilates para corredores: uma revisão da literatura. Disponível em: $<$ https://ceafi.edu.br/site/wp-content/uploads/2019/05/os-beneficios-da-pratica-dometodo-pilates-para-corredores-uma-revisao-da-literatura.pdf>Acesso em: 26 de nov. 2020.

SOUZA, Thales Rezende de. Relação entre o torque de rotação lateral do quadril e a cinemática do pé. 2012. 134 f. Tese (Doutorado em Ciências da Reabilitação). Universidade Federal de Minas Gerais, Belo Horizonte, MG, 2012.

SOUZA, Richard B. An evidence-based videotaped running biomechanics analysis. Physical medicine rehabilitation clinics, v. 27, n. 1, p. 217-236, 2016.

VANNATTA, C. Nathan; HEINERT, Becky L.; KERNOZEK, Thomas W. Biomechanical risk factors for running-related injury differ by sample population: A systematic review and metaanalysis. Clinical biomechanics, v. 75, p. 104991, 2020.

\section{Dados da primeira autora:}

Email: helenspadari28@hotmail.com

Endereço: Alameda João Dal Sasso, 800, Universitário, Bento Gonçalves, RS, CEP: 95705-266, Brasil.

Recebido em: 29/04/2021

Aprovado em: 15/07/2021

\section{Como citar este artigo:}

SPADARI, Hélen; BRODT, Guilherme Auler. A influência do nível de flexibilidade e da mobilização articular nas variáveis biomecânicas da corrida. Corpoconsciência, v. 25, n. 2, p. 162-174, mai./ ago., 2021. 